\title{
Romans de la dévastation : Le Premier Cercle d'Alexandre Soljenitsyne et La Plaisanterie de Milan Kundera
}

Marie-Odile Thirouin

\section{(2) OpenEdition \\ Journals}

Édition électronique

URL : http://journals.openedition.org/recherchestravaux/503

DOI : $10.4000 /$ recherchestravaux.503

ISSN : 1969-6434

Éditeur

UGA Éditions/Université Grenoble Alpes

Édition imprimée

Date de publication : 15 mai 2012

Pagination : $33-48$

ISBN : $978-2-84310-227-1$

ISSN : 0151-1874

Référence électronique

Marie-Odile Thirouin, «Romans de la dévastation : Le Premier Cercle d'Alexandre Soljenitsyne et La Plaisanterie de Milan Kundera », Recherches \& Travaux [En ligne], 80 | 2012, mis en ligne le 15 novembre 2013, consulté le 07 septembre 2020. URL : http://journals.openedition.org/recherchestravaux/503 DOI : https://doi.org/10.4000/recherchestravaux.503 
Marie-Odile Thirouin

Université Lumière - Lyon 2

\section{Romans de la dévastation : Le Premier Cercle d'Alexandre Soljenitsyne et La Plaisanterie de Milan Kundera}

Et je pense à Soljenitsyne. Ce grand homme était-il un grand romancier? Comment pourrais-je le savoir? Je n'ai jamais ouvert aucun de ses livres. Ses retentissantes prises de position (dont j'applaudissais le courage) me faisaient croire que je connaissais d'avance tout ce qu'il avait à dire ${ }^{\mathrm{I}}$.

Kundera s'interroge en ces termes après avoir remarqué à quel point les romanciers sont négligés par une Académie française plus friande de grands hommes que de grands écrivains. Or, le véritable romancier (il doute ici que le dissident Soljenitsyne puisse en être un) ne saurait interposer sa personne entre le public et son œuvre, c'est-à-dire faire de cette dernière la simple caisse de résonance de ses opinions, aussi justes et nobles soient-elles.

Est-on en droit, après ce jugement certes modéré dans la forme, mais réprobateur sur le fond, de proposer une comparaison entre deux romans de Soljenitsyne et de Kundera²? Oui, sans doute, d'abord parce que "pour comprendre, il faut comparer", comme l'écrit Kundera lui-même parce qu'il lui arrive aussi de comparer un roman qu'il aime avec un autre qu'il n'aime pas, à l'occasion d'une analogie thématique frappante - par

I. M. Kundera, Une rencontre, III : «Les listes noires ou divertimento en hommage à Anatole France», Gallimard, 2009, p. 69.

2. A. Soljenitsyne, Le Premier Cercle (В круге первом, dernière version révisée : 1978), Robert Laffont, coll. «Pavillons poche», 2007 (dorénavant PC), et M. Kundera, La Plaisanterie (Žert, 1967), Gallimard, coll. "Folio», 2005 (dorénavant $L P$ ).

3. M. Kundera, Le Rideau, $4^{\mathrm{e}}$ partie : "Qu'est-ce qu'un romancier?", Gallimard, 2005, p. I05. 
exemple, quand il oppose Le Procès de Kafka et 1984 d'Orwell, "qui lui aussi est l'Histoire d'une arrestation et d'un procès ${ }^{4}$. Les raisons pour lesquelles Kundera refuse alors de considérer Orwell comme un "vrai» romancier, nous intéressent ici au premier chef, car ce sont visiblement les mêmes qui expliquent ses réticences à l'encontre de Soljenitsyne : Orwell, dont Kundera disait déjà dans L'Art du roman que ce qu'il nous dit "aurait pu être dit aussi bien (ou plutôt beaucoup mieux) dans un essai ou dans un pamphlet'", nous aurait donné là non pas un roman, mais «une pensée politique déguisée en roman» (je souligne); cette pensée politique, quoique «lucide et juste», ne tirerait d'autre bénéfice de la forme romanesque que son affadissement et son gauchissement, tandis qu'en retour, cette pensée ne recevrait de la forme romanesque qu'elle adopte aucun éclairage supplémentaire ni supérieur à celui donné par la sociologie ou la politologie, comme en témoignent des situations et des personnages "d'une platitude d'affiche»; le choix du roman se justifierait-il alors, en dernière instance, par l'occasion qu'il donnerait de "vulgariser de bonnes idées»?

Non plus. Car les idées mises en roman n'agissent plus comme idées mais précisément comme roman, et dans le cas de 1984 elles agissent en tant que mauvais roman [...]. L'influence néfaste du roman d'Orwell réside dans l'implacable réduction d'une réalité à son aspect purement politique et dans la réduction de ce même aspect à ce qu'il a d'exemplairement négatif. Je refuse de pardonner cette réduction sous prétexte qu'elle était utile comme propagande dans la lutte contre le mal totalitaire. Car ce mal, c'est précisément la réduction de la vie à la politique et de la politique à la propagande. Ainsi le roman d'Orwell, malgré ses intentions, fait lui-même partie de l'esprit totalitaire, de l'esprit de propagande. Il réduit (et apprend à réduire) la vie d'une société haïe en la simple énumération de ses crimes.

C'est avec à l'esprit cette mise en garde contre l'asservissement du roman à un projet politique que je veux entamer notre comparaison : doit-on, à l'exemple de Kundera, se passer de lire Soljenitsyne? Sait-on d'avance tout ce qu'il a à nous dire et que l'on trouve maintenant exposé, avec plus d'efficacité informative, dans les ouvrages que des historiens ont consacrés à la société soviétique ou encore au goulag? Le succès de Soljenitsyne n'est-il qu'un succès de scandale lié à une conjoncture politique précise, mais périmée - celle de la lente prise de conscience par l'Occident des crimes du communisme dont l'œuvre du romancier russe ne nous fournirait que «la simple énumération»?

4. Id., Les Testaments trahis, $8^{\mathrm{e}}$ partie : «Les chemins dans le brouillard», Gallimard, coll. «Folio", I993, p. 268-269.

5. Id., L’Art du roman, $\mathrm{I}^{\text {re }}$ partie : "L'héritage décrié de Cervantès ", Gallimard, coll. "Folio", I986, p. 23.

6. Id., Les Testaments trahis, ouvr. cité, 8 partie, p. 269. 
Ces questions sont d'autant plus opportunes que La Plaisanterie, parue en France en 1968 dans le contexte du Printemps de Prague et de sa répression, a connu pour sa part «le malheur d'être perçu[e] comme une sorte de document sociologique ou de manifeste politique, c'est-à-dire comme une autre pièce à conviction dans le procès du stalinisme bureaucratique ${ }^{7}$ » : cette lecture, pourtant vigoureusement récusée par Kundera, a toujours pesé sur la réception du roman kundérien, au moins jusqu'en 1989 . Il nous appartient donc de nous en défier, tant dans le cas de La Plaisanterie que du Premier Cercle, pour ne pas transformer ces œuvres issues de ce qu'on sait maintenant avoir été une «fin d'Empire» en outils politiques appartenant désormais au passé.

Le lecteur de 2012 peut succomber à une tentation symétrique à celle de la réduction au document historique ou au pamphlet politique : une lecture de nos romans non pas tournée vers le passé cette fois, mais orientée vers l'avenir. Il est en effet tentant de chercher dans la littérature antérieure à 1989 une prophétie ou du moins le pressentiment de l'effondrement du système politique et économique instauré en Russie à partir de 1917 et en Tchécoslovaquie depuis 1948. On tomberait encore, par le biais du roman d'anticipation cette fois, sur 1984 , que Kundera oppose sur ce point aussi au Procès de Kafka. Il ne voit en effet aucun rapport entre la capacité apparente de "pré-vision" du Procès et les réalisations concrètes de l'Histoire : le roman n'obéit pas à la même temporalité que celle qui enchaîne les événements historiques et politiques, dont il se trouve parfois, de manière surprenante, qu'ils réalisent a posteriori ce que l'on trouve déjà dans la fiction :

La rencontre de l'univers réel des États totalitaires et du "poème» de Kafka gardera toujours quelque chose de mystérieux, et elle témoignera que l'acte du poète, par son essence même, est incalculable; et paradoxal : l'énorme portée sociale, politique, "prophétique» des romans de Kafka réside justement dans leur "non-engagement", c'est-à-dire dans leur autonomie totale à l'égard de tous programmes politiques, concepts idéologiques, prognoses futurologiques ${ }^{8}$.

C'est donc bien pour eux-mêmes que je veux examiner ici Le Premier Cercle et La Plaisanterie, l'un par rapport à l'autre, et non pas comme les produits instrumentalisés de considérations politiques qui leur seraient extérieures, tant de la part de leurs auteurs que de leurs lecteurs. Prenons le pari que ces deux romans ont quelque chose de singulier à nous dire sur l'existence humaine en situation de "dévastation", c'est-à-dire telle que les conditions historiques créées par l'Empire soviétique ont pu la mettre en

7. Fr. Ricard dans sa postface à La Plaisanterie (ouvr. cité), intitulée «Le roman de la dévastation", $L P$, p. 458

8. M. Kundera, L’Art du roman, ouvr. cité, $5^{\mathrm{e}}$ partie : "Quelque part là-derrière», p. I4O. 
lumière (d'abord aux yeux de nos deux romanciers, puis par leur intermédiaire à nos yeux de lecteurs). Nous admettrons en outre que leur éclairage réciproque nous permettra de mieux définir ce savoir original et les deux conceptions du roman qui le portent.

\section{La parenté de deux romans}

On le sait, Kundera déteste qu'on le compare aux romanciers russes, en dépit de son admiration pour eux, à cause de l'effet d'optique qui fait que, depuis l'Occident, on considère souvent la Russie et l'Europe centrale comme un monde culturel homogène, connu du temps de la guerre froide sous le nom d' "Europe de l'Est» ou de «monde slave"». Or il n'en est rien, bien sûr : Le Premier Cercle est issu du centre de l'Empire soviétique et La Plaisanterie de sa périphérie occidentale asservie à la suite de la Seconde Guerre mondiale. Mais en dépit de cette appartenance culturelle à deux mondes distincts, ponctuellement réunis par les hasards de la politique et de l'Histoire, on est frappé par la surprenante parenté du roman russe et du roman tchèque, sur le plan interne (thématique et structurel) comme sur le plan externe (les circonstances entourant leur genèse et leur carrière).

Commençons par cette similarité externe, la plus anecdotique. Le Premier Cercle et La Plaisanterie sont les deux premiers romans écrits par des écrivains approximativement de la même génération, soumis aux mêmes idéaux révolutionnaires auxquels ils commencent par adhérer. Soljenitsyne et Kundera, devenus romanciers après une expérience de marginalisation plus longue, radicale et violente pour le premier que pour le second, sont privés l'un et l'autre de leur public «naturel» par la censure, puis par un exil qui leur est imposé dans les années 1970 : l'Histoire éditoriale mouvementée des deux romans porte évidemment la marque de toutes ces vicissitudes et fait qu' ils ont accompagné leurs auteurs pendant au moins vingt ans ${ }^{\mathrm{I}}$. Ultime coïncidence :

9. Voir M. Kundera, Le Rideau, ouvr. cité, $2^{\mathrm{e}}$ partie : «Die Weltliteratur» («L'homme de l'Est»), p. 58-60, et le célèbre article renié (parce que purement conjoncturel) : «Un Occident kidnappé ou la tragédie de l'Europe centrale», Le Débat, n 27, novembre 1983, p. 3-22.

IO. Le Premier Cercle, écrit de 1955 à 1958 , "défiguré» dans une cinquième version vainement destinée à la publication en URSS en 1964, saisi par le KGB en 1965, récrit en 1968, paraît en russe dans cette septième version en 96 chapitres, à Paris, aux éditions YMCA-Press, en 1978, puis en français, en 1982, (les éditions russe et française de 1968 ont été publiées à Paris à partir d'une sixième version incomplète en 87 chapitres; Moscou autorise une édition complète en russe en 1990, rééditée plusieurs fois depuis). La Plaisanterie, achevée le 5 décembre 1965, parue en tchèque à Prague aux éditions Československý spisovatel en 1967 (rééditions en I968 et 1969), puis retirée de la vente, connaît une première traduction française par M. Aymonin en 1968, que Kundera révise une première fois en compagnie de 
Kundera a participé en 1968 à Prague au scénario de La Plaisanterie, admirablement adaptée et filmée par son ami Jaromil Jireš, tandis que Gleb Panfilov s'est assuré la participation de Soljenitsyne lorsqu'il a réalisé les dix épisodes du Premier Cercle diffusés par la télévision russe en 2006 (Soljenitsyne a contribué à l'élaboration du scénario et dit les textes de la voix off ${ }^{\text {II)}}$ ).

Un tel concours de circonstances justifie à lui seul qu'on rapproche les deux romans : dans un contexte historique commun, ils occupent une place charnière aux origines de la vocation romanesque de leurs auteurs qui, en outre, ont aidé à les transposer au cinéma, leur assurant ainsi une large audience auprès de leur public d'origine. Les convergences internes sont encore plus frappantes : Le Premier Cercle fait le récit de quatre journées, du samedi 24 décembre au soir, au mardi 27 décembre 1949 au matin, et est structuré par les allers et retours de la narration entre la prison-laboratoire (la charachka) de Marfino et le "dehors", Moscou, auquel touche ce "premier cercle», relativement privilégié, de l'enfer du goulag (on y est nourri correctement, on n'y fait pas de travaux de force, on n'y est pas battu par les gardiens ni mêlé aux droits communs); la narration trouve son unité au long de deux fils d'intrigue entremêlés : l'enquête confiée à notre charachka, spécialisée dans le domaine de l'acoustique, pour identifier la personne qui, le soir du 24 décembre, a appelé d'une cabine téléphonique l'ambassade américaine à Moscou en l'informant du vol de secrets nucléaires par un espion soviétique; puis la livraison des prototypes de deux appareils destinés au cryptage et au décryptage de la voix humaine, commandés par Staline qui se fait pressant : les «trois mousquetaires», Gleb Nerjine, Lev Roubine et Dimitri Sologdine, doivent décider s'ils collaborent ou non avec leurs geôliers, décision qui détermine leur sort à la fin du roman.

De son côté, La Plaisanterie raconte les trois journées du dernier weekend de mai 1965 , du vendredi soir au dimanche en fin d'après-midi, traditionnellement consacré en Moravie à un événement folklorique, musical et poétique, la Chevauchée des Rois. À l'occasion de cette fête, l'ensemble des protagonistes du roman se trouve réuni dans la ville natale de Ludvik, le personnage principal. Les allers et retours de la narration ne se font pas ici dans

C. Courtot en I980, puis une nouvelle fois, seul, en 1985 (version française définitive valant original), tandis que le public tchèque dispose d'une édition aux Sixty-Eight Publishers de Toronto de 1989 et d'une version définitive, aux éditions Atlantis de Brno, de 1990 également (rééditions en 1996 et 2007).

II. Le film de J. Jireš (1935-200I), Žert, est diffusé en France en DVD par la société de distribution Malavida Films, et les dix épisodes du film de G. Panfilov (né en 1934) sont visibles en russe sur le site <http://www.livekino.am/v_kruge_pervom-serial.aspx> (pour divers documents concernant ce film, voir aussi le site <http://www.vkrugepervom.ru/content. html? $\mathrm{cid}=42 \& \mathrm{p}=2>$ ). 
l'espace ${ }^{12}$, mais dans le temps, avec les années 1948-1956 : ce sont les années du stalinisme tchécoslovaque et celles de la marginalisation de Ludvik à la suite d'une "mauvaise plaisanterie ${ }^{13}$ » (une carte postale provocante, adressée en août 1949 à la jeune fille convoitée par Ludvik, est interceptée et rendue publique, Ludvik exclu du parti et de la faculté des sciences, envoyé dans un bataillon disciplinaire en Moravie-Silésie où il travaille cinq ans dans les mines et fait un an de prison pour désertion, avant de rentrer à Prague où il reprend discrètement ses études interrompues). En ces jours de mai 1965, il nourrit le projet de se venger de Pavel, celui qu'il considère comme le principal responsable de son malheur.

\section{Polyphonies romanesques}

Cette très forte unité spatiale et temporelle encadrant une action resserrée, à un ou deux fils de trame, crée bien sûr un effet de loupe et produit un sentiment de crise, d'urgence. Elle est associée, en revanche, dans les deux romans à une architecture globale extrêmement complexe, organisée selon des principes à la fois mathématiques et musicaux (est-ce un hasard si les deux personnages principaux du Premier Cercle et de La Plaisanterie, Nerjine et Ludvik, sont des mathématiciens ${ }^{\mathrm{I} 4}$ ?). "C'est le matériau qui dicte", dit Soljenitsyne en 1983 quand on l'interroge sur ses choix formels ${ }^{15}$ : or, il ne se sent pas à l'aise, nous dit Georges Nivat, dans une œuvre où il y a trop d'espace et par conséquent, organise ce matériau en courts chapitres autonomes, selon «un besoin "mathématique" de faire passer les plans innombrables de la réalité par des "nœuds" »; mais surtout, ce goût pour la «forme dense» lui est dicté par des conditions d'écriture difficiles, en cachette, dans l'urgence : "chaque chapitre est comme écrit face à la mort, dans la fièvre et la sérénité d'une finalité intensément ressenti $e^{16}{ }$. L'unité primordiale du chapitre est ensuite saisie dans une forme globale, "polyphonique» (car on y entend, au gré des chapitres, les voix du narrateur et de ses nombreux personnages, modulées dans

I2. Le village de Vlčnov, au centre de cet événement, constitue la banlieue de la ville natale de Ludvik dans laquelle on peut reconnaître, à l'aide du film de J. Jireš, Uherské Hradiště. L'information n'est pas nécessaire à la compréhension du roman, même si elle contribue à lui donner sa couleur "locale», morave, en réalité très marquée par opposition au monde pragois.

13. Expression employée dans $L P$, p. 366, 408, 4I4.

I4. $P C$, p. 47 et $L P$, p. 8I.

15. G. Nivat, Le Phénomène Soljenitsyne, Fayard, 2009, p. 236. G. Nivat indique la date de 1977, mais l'interview de Soljenitsyne par B. Pivot, à laquelle il pense sans doute, est de 1983. 16. Ibid., p. 240. 
des tonalités diverses colorées par la peur, la colère, l'ironie, la souffrance, l'humour...), forme qui culmine à son tour dans quelques "clefs de voûte» lyriques et contemplatives ${ }^{17}$, comme des pauses au cours desquelles le lecteur est emporté au-dessus de la narration et peut en méditer l'architecture à son aise. C'est encore Georges Nivat qui fait remarquer que la première édition russe du Premier Cercle s'ouvre sur une liste de titres de chapitres sans pagination, organisés selon l'axe médian de la page, titres qui disparaissent dans le corps du texte (les 96 chapitres sont simplement numérotés, sauf la nouvelle intitulée "Le sourire de Bouddha») mais se lisent comme un poème ou "une ouverture musicale [annonçant] les répons d'un chapitre à l'autre ${ }^{18}$ ".

Les principes qui président à la composition des romans de Kundera, qu'il a abondamment développés dans ses essais, sont mieux connus. Rien n’y est laissé au hasard, on le sait :

La division du roman en parties, des parties en chapitres, des chapitres en paragraphes, autrement dit l'articulation du roman, je la veux d'une très grande clarté. Chacune des sept parties est un tout en soi. Chacune est caractérisée par son propre mode de narration [...]. Chacune a sa propre perspective (est racontée du point de vue d'un autre ego imaginaire). Chacune a sa propre longueur: ordre de ces longueurs dans La Plaisanterie : très courte; très courte; longue; courte; longue; courte; longue. [...] Les chapitres, eux aussi, je veux qu'ils soient, chacun, un petit tout en soi $^{19}$.

La plus petite unité autonome ou discrète est donc ici celle du paragraphe, mais les chapitres, numérotés et séparés nettement les uns des autres, déterminent à leur tour par leur nombre le tempo singulier des parties, comparables alors aux différents mouvements d'une composition musicale avec leur "atmosphère émotionnelle " particulière : Kundera utilise à plusieurs reprises cette métaphore musicale à propos de ses romans ou parle encore d' "ordre » ou de «structure mathématique», par exemple à propos du volume respectif attribué à chacune des quatre voix narratives de La Plaisanterie selon un rapport qui «n'est pas quelque chose de calculé», mais constitue chez lui "une obsession ». Ce souci extrême à la fois de la diversité, de la spécificité et de la distribution sonores des unités formellement définies est lié sans doute, comme déjà chez Soljenitsyne, à la culture musicale de l'auteur, et correspond, comme chez Soljenitsyne encore, à la nécessité de faire entendre des voix différentes - sauf qu'ici, à la différence de Soljenitsyne cette fois, la polyphonie n'est pas collecte ni collection de voix en réaction à leur effacement programmé, mais

17. Ibid., p. 239.

I8. Ibid., p. 237. Cette liste n'apparaît pas dans l'édition française.

19. M. Kundera, L'Art du roman, ouvr. cité, $4^{\mathrm{e}}$ partie : "Entretien sur l'art de la composition", p. I07-IO8. Les fragments de citations qui suivent sont extraits de ce même texte, p. IO6-II2. 
vise à relativiser les discours dont elles sont porteuses, pour qu'aucun ne puisse prétendre au statut de "Vérité» du roman. Car :

[...] le roman est incompatible avec l'univers totalitaire. Cette incompatibilité [...] est non seulement politique ou morale mais ontologique. Cela veut dire : le monde basé sur une seule Vérité et le monde ambigu et relatif du roman sont pétris chacun d'une manière totalement différente. La Vérité totalitaire exclut la relativité, le doute, l'interrogation et elle ne peut donc jamais se concilier avec ce que j'appellerais l'esprit du roman ${ }^{20}$.

On voit ce que la pratique «cumulative» de la polyphonie chez Soljenitsyne et celle, «soustractive» ou " corrective» de Kundera, - deux variantes du traitement romanesque auquel nos auteurs soumettent leur «matériau» - doit à une situation existentielle, celle créée par l'«univers totalitaire» univoque : elles sont la transposition esthétique, ressentie comme nécessaire, d'un souci d'individuation (rendre à chacun sa voix) qui tend certes dans deux directions différentes, mais qui a des raisons "ontologiques", non strictement matérielles ou intellectuelles. Car le roman, art par excellence des Temps modernes qui «ont fait de l'homme, de l'individu, d'un ego pensant, le fondement de tout ${ }^{21}$ ", est ainsi «naturellement» la forme susceptible d'accueillir la diversité des voix sans les écraser, de les faire entendre, toutes ou à plusieurs, sans donner la primauté à l'une plutôt qu'à l'autre, chacune ayant sa "valeur».

Voici donc ce que permet la littérature plutôt que l'essai ou le pamphlet : rendre compte de la pluralité des voix, montrer sans démontrer. Reste à comprendre ce que les romans qui portent ces voix jusqu'à nous ont à nous dire qu'eux seuls peuvent dire, non pas sur l'Histoire ni sur la société dans l'Empire soviétique de l'après-guerre, mais sur l'homme dans les conditions de cet empire à ce moment de son Histoire. En d'autres termes, quel savoir anthropologique singulier nous est dévoilé par ces deux romans? Est-ce le même? Pourquoi donne-t-il lieu à deux pratiques aussi différentes de la polyphonie romanesque?

\section{L'homme dans le monde dévasté chez Kundera}

Il faut aborder ici le dernier plan où convergent Le Premier Cercle et La Plaisanterie, celui du thème, au sens d' "interrogation existentielle» ou de "questionnement existentiel ${ }^{22}$ » : la dévastation. François Ricard, dans sa postface à

20. Ibid., $\mathrm{I}^{\mathrm{re}}$ partie : "L'héritage décrié de Cervantes», p. 25.

2I. M. Kundera, Les Testaments trahis, ouvr. cité, $9^{\mathrm{e}}$ partie : «Là, vous n'êtes pas chez vous, mon cher", p. 323.

22. Voir M. Kundera, L'Art du roman, ouvr. cité, p. IO4 et Les Testaments trahis, ouvr. cité, p. 200. 
La Plaisanterie de 2003, fait déjà de ce mot thème la clé du roman qu'il qualifie globalement de "roman de la dévastation ${ }^{23}$ ", formule que je lui emprunte en l'appliquant aussi au roman de Soljenitsyne. Il me semble toutefois que, par crainte de voir le lecteur réduire cette dévastation à la seule sphère politique, François Ricard se hâte de lui donner un sens abstrait, métaphysique (c'est l'expérience de l'«universelle plaisanterie», du "dérèglement» et de la "suspension des significations $\left.{ }^{24} »\right)$, certes juste, mais à son tour trop étroit. Car la dévastation renvoie moins à la question du sens qu'à la question de la valeur des choses : elle est en premier lieu "pillage», au sens matériel du terme. Pillage des paysages (que l'on pense aux vues d'Ostrava et des bords de la Morava dans $\mathrm{La}$ Plaisanterie, à la poussière qui couvre la ville natale de Ludvik, signe concret de la dévastation ${ }^{25}$ ), pillage des vies aussi, comme celles de Lucie et de Ludvik :

[...] les histoires de nos deux vies étaient fraternelles et conjointes, étant toutes les deux histoires de dévastation; ainsi qu'on avait en Lucie dévasté l'amour charnel et privé son existence d'une valeur élémentaire, ma vie aussi fut spoliée des valeurs sur lesquelles elle voulait s'appuyer ${ }^{26}$.

Mais Jaroslav, Helena et Kostka également, les trois autres voix narratives de La Plaisanterie à côté de Ludvik, font l'expérience de ce pillage, c'est-àdire de la frustration, de la privation soudaine et violente de ce en quoi ils croyaient et dont ils découvrent que cela n'a aucun prix pour personne, ni matériel, ni symbolique : «tous ces univers personnels sont saisis au moment de leur décomposition", écrit Kundera en ajoutant une variation au thème de la dévastation ${ }^{27}$. Or, nul ne peut être rendu coupable de la perte générale des valeurs : c'est ce qu'apprend Ludvik avec retard, à la fin de "cette âcre journée » de mai 1965, lorsqu'il comprend la vanité qu'il y a à vouloir se venger de l'offense subie quinze ans plus tôt :

Oui, j'y voyais clair soudain : la plupart des gens s'adonnent au mirage d'une double croyance : ils croient à la pérennité de la mémoire (des hommes, des choses, des actes, des nations) et à la possibilité de réparer (des actes, des erreurs, des péchés, des torts). L'une est aussi fausse que l'autre. La vérité se situe juste à l'opposé : tout sera oublié et rien ne sera réparé. Le rôle de la réparation (et par

23. LP, p. 457. Outre les mots "dévastation" et "dévaster" qui font l'objet de répétitions frappantes, on trouve dans La Plaisanterie nombre de termes ou d'expressions qui en constituent des variations : ravage, saccage, gâchis, défaite, ruine, désolation, échec, destruction, monde en miettes...

24. $L P$, p. 48I, 469, 485 .

25. $L P$, p. 47 et I07.

26. $L P$, p. 448.

27. M. Kundera, Les Testaments trahis, ouvr. cité, $\mathrm{I}^{\mathrm{re}}$ partie : «Le jour où Panurge ne fera plus rire", p. 22. 
la vengeance et par le pardon) sera tenu par l'oubli. Personne ne réparera les torts commis, mais tous les torts seront oubliés ${ }^{28}$.

La vérité existentielle à laquelle parvient ici Ludvik, c'est que rien ne vaut qu'on s'y attache, c'est-à-dire ne mérite d'être érigé en valeur (positive ou négative) fixée par sa croyance et orientant son existence, car toute valeur est vouée, dans le déroulement erratique du temps, à l'oubli, à la disparition, au non-être - c'est en quelque sorte sa condition "naturelle». Ce savoir est l'aboutissement de "l'expérience anthropologique fondamentale» de perte et de privation que fait Ludvik en 1949, lorsqu'il se voit, du jour au lendemain, rejeté par ses pairs, professeurs et camarades d'études, au cours d'un pseudo-procès dont il tire cette définition de l'homme : "un être capable dans n'importe quelle situation d'envoyer son prochain à la mort ${ }^{29}$ ".

L'expérience de la dévastation n'a pourtant rien d'une tragédie, François Ricard a raison de le souligner en écrivant que «le sujet dévasté [...] est comme délivré, abandonné à une légèreté à la fois libératrice et vaine ${ }^{30}$ ", pleinement rendu à lui-même, individu sans lien ni responsabilité envers rien ni personne. Et d'ailleurs, c'est au moment où Ludvik se constitue en sujet individué qu'il retrouve la possibilité d'établir un lien gratuit, éphémère, immatériel avec autrui et accepte de jouer dans l'orchestre désuet de Jaroslav, réuni autour d'un objet dénué de toute valeur et comme inexistant : la musique folklorique morave, soudain dotée d'une beauté "à contresens", puisque personne ne veut d'elle, personne ne la convoite, personne ne l'entend même dans la cacophonie ambiante - les musiciens jouent "pour le plaisir», isolés provisoirement de tous et à l'abri dans une sorte de «cabine de verre suspendue». Et la "pitié», la "compassion» pour cet objet délaissé, qui ne saurait donc se constituer en valeur, poussent à nouveau Ludvik, contre toute attente, vers les autres - ce petit groupe de quelques musiciens. Il n'est bien sûr pas anodin qu'une telle expérience fugace et paradoxale de bonheur partagé, au milieu des ruines de toutes les valeurs, produise un plaisir de nature esthétique : le sentiment de "beauté» est en effet la signature, chez Kundera, non pas d'un moment où le sens est suspendu, mais d'un moment de grâce, où l'on est comme autorisé à adhérer, à croire à quelque chose et donc à rejoindre autrui - à suspendre l'exercice systématique de l'incroyance ${ }^{31}$.

28. $L P$, p. 422.

29. M. Kundera, L'Art du roman, ouvr. cité, $2^{\mathrm{e}}$ partie : "Entretien sur l'art du roman", p. $5 \mathrm{I}$.

30. $L P$, p. 48I.

3I. Les citations sont tirées de l'épisode narré dans $L P$, p. 447-453. 


\section{Une autre conception du roman, celle de l'ancien zek}

Dans Le Premier Cercle, comme dans La Plaisanterie, la dévastation est d'abord concrète, le produit du monde totalitaire qui réunit les conditions historiques de sa manifestation. Soljenitsyne consacre, il est vrai, plus de temps à en décrire les effets que Kundera, qui «stylise» et ne garde du contexte que "quelques objets indispensables à l'action» et particulièrement révélateurs, même si du point de vue de l'historien, ils paraissent d'un intérêt mineur ${ }^{32}$. Mais la richesse du tableau que fait Soljenitsyne de la société soviétique ne vise pas plus à la dénonciation de celle-ci qu'elle ne correspond à un désir documentaire ou réaliste gratuit, étranger à la narration : elle procède d'un besoin d'exhaustivité doublé d'une "passion de comprendre l'objectivité du monde», pour citer encore Kundera ${ }^{33}$. Les éléments issus du contexte historique et politique ne sont d'ailleurs jamais donnés à part, comme des coulisses, mais toujours en rapport avec un personnage, son histoire, une situation singulière qui le place dans l'éclairage adéquat pour que le lecteur puisse comprendre son "code existentiel», entendre sa voix dans la couleur qui lui est propre - par exemple celles d'Innokenti Volodine et de Clara Makaryguine se promenant dans la campagne collectivisée de Rojdestvo-surIstia, par une «terre mutilée, défigurée, dolente ${ }^{34}$ ".

La particularité de l'art de Soljenitsyne tient d'une part au foisonnement de ces personnages dont on découvre toujours de nouveaux jusqu'aux dernières pages du roman, et d'autre part au fait que celui-ci mêle personnages historiques, personnages réels et personnages de fiction : «la charachka de Marfino

32. "Voici quelques principes qui sont les miens. Premièrement : toutes les circonstances historiques, je les traite avec une économie maximale. Je me comporte à l'égard de l'Histoire comme le scénographe qui arrange une scène abstraite avec quelques objets indispensables à l'action. Deuxième principe : parmi les circonstances historiques je ne retiens que celles qui créent pour mes personnages une situation existentielle révélatrice. Exemple : dans La Plaisanterie, Ludvik voit tous ses amis et condisciples lever la main pour voter, avec une totale facilité, son exclusion de l'université et faire ainsi basculer sa vie. Il est sûr qu'ils auraient été capables, si nécessaire, de voter avec la même facilité sa pendaison. D’où sa définition de l'homme : un être capable dans n'importe quelle situation d'envoyer son prochain à la mort. L'expérience anthropologique fondamentale de Ludvik a donc des racines historiques, mais la description de l'Histoire elle-même (le rôle du Parti, les racines politiques de la terreur, l'organisation des institutions sociales, etc.) ne m'intéresse pas et vous ne la trouverez pas dans le roman. Troisième principe : l'historiographie écrit l'histoire de la société, non pas celle de l'homme. C'est pourquoi les événements historiques dont mes romans parlent sont souvent oubliés par l'historiographie [...], mais d'une signification anthropologique suprême!» (M. Kundera, L'Art du roman, ouvr. cité, $2^{\mathrm{e}}$ partie "Entretien sur l'art du roman », p. 50-5I )

33. Ibid., $6^{\mathrm{e}}$ partie : «Soixante-treize mots», p. 165.

34. PC, p. 403-426. 
et presque tous ses habitants ont été peints d'après nature», nous dit la note finale sur la rédaction et l'édition du Premier Cercle 35 qui est d'ailleurs dédié «à mes amis de la charachka». On peut bien sûr confondre cet ancrage manifeste dans la réalité avec un souci de documentation historique ou un projet politique de dénonciation quelconque, mais ce serait faire injure au roman dont l'ambition est d'ouvrir ses pages à une réalité humaine niée, donc invisible et inaudible: Soljenitsyne n'est pas le porte-parole des idées d'autrui ni de ses propres idées, mais il prête sa voix au «tocsin muet : volées vibrantes de cloches, gémissements, cris, appels, hurlements à la mort, qu'un vent constant, têtu, emport[e] loin des oreilles humaines ${ }^{36}{ }^{\prime}$. Dans cette situation, le roman est précisément la forme qui lui permet de tout montrer d'une réalité qu'on ne veut ou ne peut pas voir, sans avoir à juger ni prendre parti (car Soljenitsyne a bien sûr un avis!), à une distance qui évite le pathos du pamphlet comme l'objectivité froide du document, qui met la voix du narrateur (celle de Nerjine-Soljenitsyne) à égalité avec toutes les autres voix. Kundera a raison : Soljenitsyne obéit à un projet qui n'est pas strictement esthétique, mais moral; pourtant il n'est pas au service d'idées, mais de personnes, ses codétenus, l'expérience partagée de la prison légitimant son projet de romancier. C'est par Soljenitsyne et par le roman que le monde entend parler et voit le goulag pour la première fois, lieu où il a achevé de se charger de "ce [double] fardeau que personne ne lui avait confié», comprendre et faire savoir :

recueillir les particules d'une chaleur encore présente pour ressusciter le cadavre [de la Russie] et montrer à tous ce qu'il avait été, et défaire sa fausse image. [...] Tôt apparue, cette insatiable envie de débusquer le mensonge de l'Histoire n'avait fait que croître [...]. Et en lui, invincible, s'était enracinée la décision de savoir et de comprendre! De mettre au grand jour! De rafraîchir les mémoires ${ }^{37}$ !

\section{«Retournement optique»: l'homme dans le monde dévasté chez Soljenitsyne}

Du point de vue interne à la narration, la peinture du contexte historique par le biais des personnages permet au romancier d'opposer avec efficacité le monde de la prison et le monde «libre». Ce faisant, Soljenitsyne s'inscrit dans

$$
\text { 35. PC, p. 991. }
$$

36. $P C$, p. 344-345. La métaphore clé du "tocsin muet» est inspirée à Soljenitsyne par le roman de Hugo consacré à la Terreur, Quatre-vingt-treize ( ${ }^{\mathrm{re}}$ partie, livre IV, chap. II : "Aures habet, et non audiet»), où le marquis de Lantenac, fraîchement débarqué en France, du haut d'une dune, voit les cloches des villages sonner le tocsin sans les entendre, à cause de l'éloignement et du vent.

37. PC, p. 345-346. 
la tradition russe du récit carcéral et il se souvient tant du Tchékhov de L'̂̂le de Sakhaline (1894) que du Tolstoï de Résurrection (1899) pour montrer en quoi le bagne soviétique se distingue des formes antérieures de détention en Russie. Le goulag est précisément la partie immergée, cachée d'un régime " totalitaire» dans la mesure où la dévastation qu'il produit s'étend à tout l'homme, à tous les domaines de sa vie physique, psychique, morale, intellectuelle, spirituelle. Empruntant cependant à Tolstoï cet art du «retournement optique» qui fait qu'Anna Karénine ne sait plus si le train où elle se trouve avance ou recule, que, dans Ivan Ilitch, on ne sait plus si l'on monte ou si l'on descend dans le brouillard ${ }^{38}$, Soljenitsyne fait de la prison le lieu de la liberté et, du monde libre, le lieu de la véritable prison. Il ne s'agit pas là d'une simple pirouette, on le devine, mais d'une conversion du regard arrachée dans la douleur. Car en étant aspiré dans le monde du goulag, Nerjine a tout perdu : «la science qui était la sienne, son temps, sa vie et même son amour pour sa femme ${ }^{39}$ ". D'ailleurs, la prison ne nous est nullement dépeinte comme un lieu idyllique : le temps y est vide, sans bords, la durée du châtiment ayant peu de rapport avec la nature mystérieuse du "crime» et pouvant s'allonger à l'infini - alors que le Raskolnikov de Dostoïevski se trouve encore dans la situation où «la faute cherche le châtiment ", pour Josef K. comme pour Ludvik, Nerjine et ses compagnons, c'est "le châtiment [qui] cherche la faute ${ }^{40}$ » les détenus subissent les formes traditionnelles de privations affectives et de "désespoir sexuel », à quoi s'ajoute la culpabilité de savoir que leurs femmes, à l'extérieur, mènent une existence de parias; la promiscuité est ici de règle, la vie en «troupeau » dans des espaces confinés d'où toute intimité est bannie ou exposée aux mouchards. Et pourtant, «la prison est une bénédiction autant qu'elle est une malédiction ${ }^{41} »$ : c'est la conviction à laquelle parvient Nerjine qui a appris à «tirer parti de la prison, [y] naitre à une vie nouvelle ${ }^{42}$ ».

Le sentiment de dévastation auquel il succombe lors de sa première année de détention (il en est à la cinquième) s'est donc converti en sentiment de bonheur :

38. Voir les pages que G. Nivat consacre au procédé si remarquable qu’il désigne sous le terme de "retournement optique», dans la préface de Résurrection de Tolstoï, Gallimard, coll. «Folio», 1994, p. I2-I6.

39. PC, p. 347.

40. M. Kundera, L'Art du roman, ouvr. cité, $4^{\mathrm{e}}$ partie : "Une phrase», p. I25. Soljenitsyne compare explicitement le bagne vu par Dostoïevski et celui qu'il a connu dans $P C$, p. 945, lors d'une de ces pauses lyriques qui sont les clés de voûte du récit (ici «la prière du zek»).

4I. $P C$, p. 244.

42. $P C$, p. 67. Voir aussi $P C$, p. 503-504: "Chaque homme a son temps à lui, où il s'est révélé pleinement, où il a senti les choses le plus profondément, où il s'est intégralement avoué à lui-même et aux autres. [...] Pour Nerjine, c'était la prison.» 
[...] au camp et surtout ici, à la charachka, le miracle se produit parfois et c'est un dimanche bien tranquille, sans corvées, où l'âme prend le temps de se dégeler, de revenir à elle. Même si rien n’a changé en mieux dans notre condition, il suffit que le joug de la prison se fasse un peu moins sentir, qu'on puisse parler à cœur ouvert, ou bien qu'on lise une page qui ne ment pas, pour se retrouver au sommet de la vague! Il y a longtemps qu'on ne vit plus vraiment, mais on n'y pense plus! C'est un état d'apesanteur, d'envol, on se découvre immatériel! [...] Il n'est pas de président ni de Premier ministre qui puisse s'endormir aussi heureux que je peux l'être du dimanche qui vient alors de s'écouler ${ }^{43}$.

L'expérience rapportée ici est l'inverse de celle que fait Ludvik, écrasé sous «le poids du vide» de sa vie une fois qu'il a été rejeté hors du chemin de l'Histoire, qu'il est devenu lui aussi invisible et a perdu la maîtrise de son "destin». Chez Soljenitsyne, la prison est certes une école de désillusion permanente, comme chez Kundera qui fait découvrir à Ludvik toute la vanité de la soi-disant "solidarité» des prisonniers; mais Nerjine, lui, ressent la prison aussi comme un lieu d'allégement et de libération - sur le plan matériel et surtout sur le plan spirituel : on y est en effet libéré de la crainte de perdre ce qu'on a déjà perdu (ceux du "dehors» ont encore tout à perdre de leur bien-être matériel ${ }^{44}$ ) et donc à même de "se faire une âme» en réfléchissant, en débattant avec ses amis - c'est cette liberté de penser qui manque le plus à Volodine lorsqu'il songe qu' «il avait connu bien des bonheurs matériels! jamais le bonheur inestimable : la liberté de dire ce qu'on pense, la liberté de commerce avec ses pairs en esprit ${ }^{45}$ ». C'est donc à la découverte de la philia, définie par Aristote comme choix réfléchi de vivre ensemble, lien désintéressé poussant l'homme à vivre avec son prochain, lieu en somme de constitution de l'humanité, que conduit la vie en prison. Nerjine l'affirme en portant un toast aux six autres convives de son "banquet» d'anniversaire :

Soyons justes! Tout n'est pas si noir dans notre condition! Ce bonheur-ci, justement : cette tablée d'hommes affranchis des contraintes, pareils aux lycéens

43. PC, p. 66.

44. C'est ce qu'explique le détenu Bobynine au puissant ministre Abakoumov : "Vous devriez comprendre et faire savoir à qui de droit, là-haut, que vous n'êtes forts que dans la mesure où vous ne prenez pas tout. L'homme à qui vous avez tout pris n'est plus sous votre coupe, il est libre.» (PC, p. I38) Ces propos sont d'ailleurs tempérés quelques pages plus loin, quand Bobynine regrette de ne pas avoir pris les cigarettes offertes par le ministre, pour lui signifier son détachement.

45. PC, p. 923. Voir aussi les propos de Tchelnov : «Le professeur Tchelnov affirmait d'ailleurs qu'il fallait employer le mot d'âme avec circonspection puisque seul le zek était assuré d'en posséder une qui fût immortelle, alors que l'homme libre, éminemment futile, s'en trouvait dépourvu» ( $P C$, p. 295) et de Guerassimovitch : «Les gens libres ne possèdent pas cette âme immortelle que les zeks conquièrent au prix de condamnations interminables, les gens libres jouissent avec voracité et maladresse de la liberté qui leur est octroyée et sont tous empêtrés dans des rêves indigents et des démarches futiles.» $(P C$, p. 838$)$ 
du temps de Pouchkine, cet échange de pensées libres, sans crainte ni dissimulation - mais c'est un bonheur que nous ne connaissions pas avant d'être prisonniers! [...] D’après la loi des sinusoïdes, je connaîtrai encore des bouffées de succès creux et de fausse grandeur. Mais je vous jure que je n'oublierai pas l'authentique grandeur de l'homme telle qu'elle sést révélée à moi en prison! [...] Levons nos verres à cette amitié qui fleurit au fond des caveaux de prison ${ }^{46}$.

Alors que Ludvik accède au sentiment de liberté quand il a coupé tous les liens pour se constituer en sujet entièrement individué ${ }^{47}$ (il ne retrouve son ami Jaroslav que pour l'emporter, terrassé, à travers la foule jusqu'à une "ambulance» - dernier mot et ultime ironie de La Plaisanterie), Nerjine l'éprouve quand la prison, de manière inattendue, lui offre l'amitié - un lien gratuit, consenti, confiant avec autrui, noyau premier d'une société civilisée où l'on réfléchit ensemble à ce qu'est l'homme, la vie, le bonheur : il gagne ainsi une "révélation essentielle sur l'existence ${ }^{48}$ », une connaissance "des profondeurs secrètes de la vie ${ }^{49} »$. La prison, pour Nerjine-Soljenitsyne, est le lieu où l'on devient homme soi-même, où l'on se constitue en sujet dans la rencontre avec son prochain - car on n'est jamais libre tout seul. C'est le sens de la métaphore à laquelle est consacré tout le chapitre 53 , au centre du Premier Cercle : la prison, à l'abri du monde que, dans un retournement éloquent, elle ne voit pas et n'entend pas à son tour, donc plongée dans le néant, est comparée à l'arche de la Bible (le mot «ковчег» est répété quatre fois) qui emporte cette société des derniers Justes, ultime reste d'humanité projeté à son sommet dans un monde dévasté et englouti par le Déluge :

Les matelots de l'arche étaient impondérables ainsi que leurs pensées. Ils n'avaient pas faim, sans être pour autant repus. N'ayant point de bonheur, ils ignoraient toute angoisse de le perdre. Leur esprit n'était hanté d'aucune préoccupation platement professionnelle, intrigue ou avancement, leurs épaules ne ployaient pas sous les soucis de logement, de combustible, du pain, des vêtements pour les enfants. L'amour, perpétuelle jouissance et tourment des hommes, ne pouvait leur communiquer ses frissons ni son agonie. La peine à purger était si longue qu'aucun d'entre eux ne songeait aux années qui suivraient sa libération. Des hommes à l'esprit distingué et cultivé, riches d'expérience mais trop requis par leur vie de famille pour offrir assez d'eux-mêmes à l'amitié, n'appartenaient plus ici qu'à leurs amis ${ }^{5^{\circ}}$.

46. $P C$, p. 525 (je souligne). Ce «banquet» fait pendant, dans le roman, à la soirée de gala chez les Makaryguine dont chaque convive doit surveiller ses propos, et constitue en outre, sur le plan thématique, une variation sur le Banquet de Platon.

47. M. Kundera, L'Art du roman, ouvr. cité, p. 192 : «Mais c’est précisément en perdant la certitude de la vérité et le consentement unanime des autres que l'homme devient individu. »

48. $P C$, p. 396.

49. $P C$, p. 244.

50. $P C$, p. 5I2-5I3. 
Grandeur ou misère de l'homme aux prises avec l'Histoire devenue folle? Il ne s'agit pas ici de choisir entre Soljenitsyne et Kundera, entre Le Premier Cercle et La Plaisanterie, mais de les lire conjointement. Ces deux romans, arrivés ensemble à la connaissance de l'Occident en 1968, à un moment qui a correspondu - on le sait maintenant - au début de la décomposition de l'Empire soviétique et symétriquement à la fin de la sympathie inconditionnelle dont ce dernier jouissait encore souvent à l'Ouest depuis la Seconde Guerre mondiale, ont été lus comme des brûlots antisoviétiques qu'ils ne sont que dans ce contexte d'affrontement et de désinformation. Vus d'aujourd'hui, ils nous montrent deux visages de l'homme confronté à «l'universelle plaisanterie» de l'Histoire, telle qu'elle se joue sur une scène qui concerne aussi bien le monde totalitaire que le monde démocratique. Kundera explore les voies par où l'homme s'arrache à l'emprise meurtrière et aveugle de l'Histoire; pour Soljenitsyne, on reste dans l'Histoire sans pouvoir connaître le terme de souffrances dont les détenus de Marfino ont seulement la conviction intime qu'elles cesseront, peut-être sans eux : «-Ma Russie... oh, ma vie... jusqu'à quand ce tourment?» demande Nerjine; et Sologdine : "Verrons-nous jamais le temps où les hommes pourront se retrouver et parler?»; et Guerassimovitch : «Bien sûr qu’ils périront. Mais nous périrons avant ${ }^{5 \mathrm{I}}$.» Car le mal finit par s'autodétruire : c'est là la seule loi qui commande l'Histoire.

5I. $P C$, p. $339,248,883$. 